\title{
Advances in neurosurgery
}

\section{Five new things}

\author{
Marlin Dustin Richardson, MD \\ Jonathon J. Parker, BS \\ Allen Waziri, MD
}

\section{Summary}

Surgical options for disease of the nervous system continue to expand in breadth and scope. These advances have been related in large part to progress in technology, translational application of molecular biology, and increasing understanding of the physiologic processes associated with neurologic disease. The current review will outline recent neurosurgical advances in the management of brain tumors, movement disorders, spinal degenerative disease, and neurologic injury. In addition, we include a brief discussion of exciting data from recent trials focusing on the brain-machine interface.

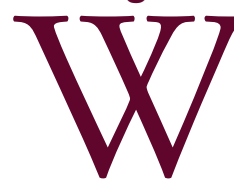

Increasing extent of tumor resection through fluorescence-guided surgery

ith the invention of CT imaging in the early 1970s, detailed brain tumor localization allowed for much more effective preoperative planning and tumor targeting. The subsequent development of MRI led to further advances in preoperative diagnosis and improvements in the ability to delineate tumor from surrounding anatomic structures. During the 1990s, intraoperative neuronavigation was developed, allowing for relatively accurate and highly convenient 3-dimensional guidance based on preoperative CT and MRI. However, while

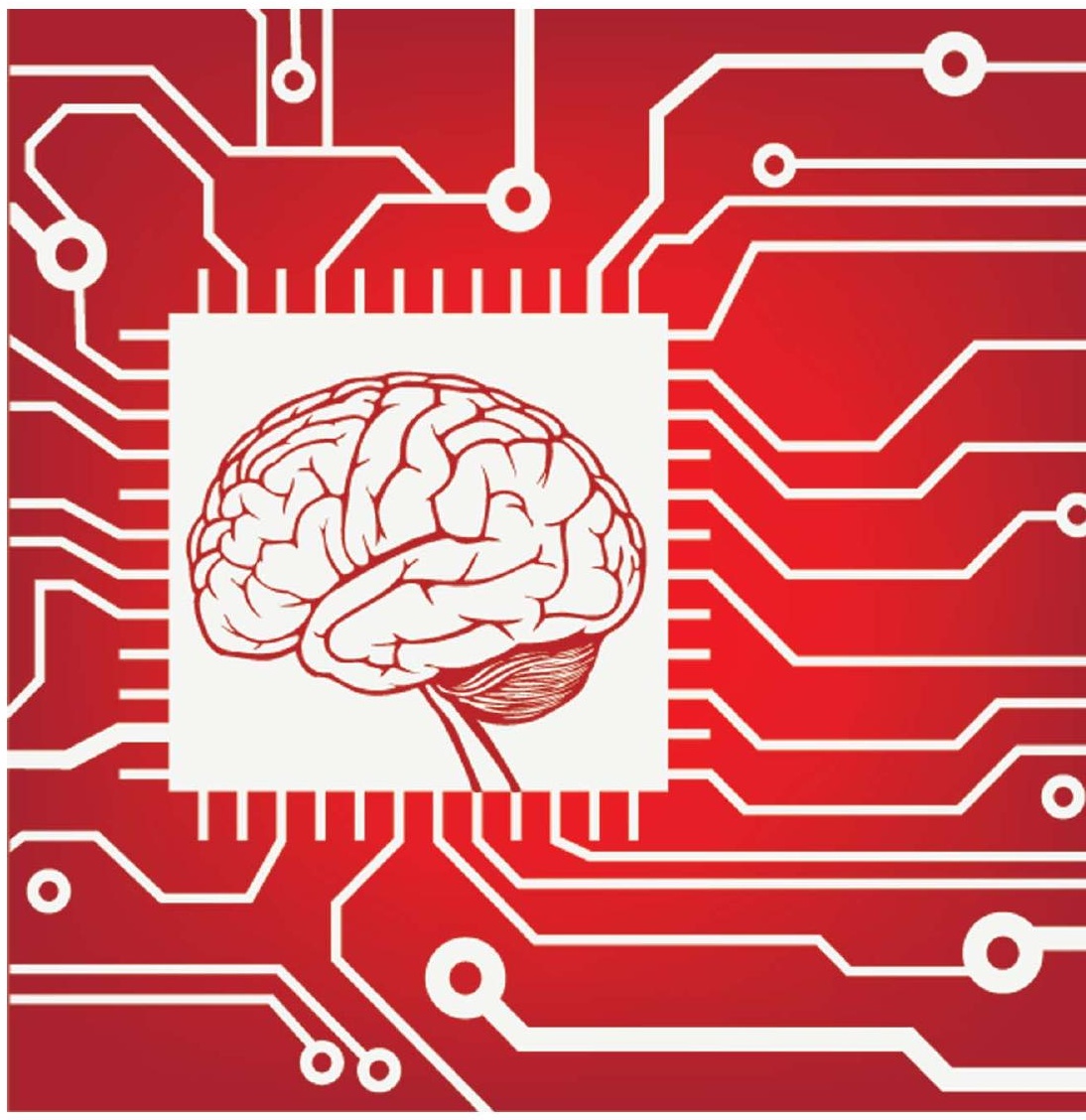
neuroimaging and neuronavigation have led to an increased ability for the neurosurgeon to define tumor borders during the approach and initial portions of the procedure, intraoperative brain deformation (secondary to drainage of CSF, effects of anesthetic manipulation, and ongoing tumor debulking) renders conventional neuronavigation inevitably inaccurate for guiding resection of infiltrating and deep tumor margins. Due to the high degree of similarity in gross appearance between intrinsic tumors and surrounding brain tissue, as well as potential apposition of tumor to eloquent brain structures, the ability to specifically visualize neoplastic tissue within the intraoperative setting is of critical importance.

Efforts to maximize surgical resection of brain tumors have recently been redoubled, as it has become widely known that extent of resection in glioblastoma correlates with increased patient

Department of Neurosurgery, University of Colorado School of Medicine, Aurora.

Correspondence to: allen.waziri@ucdenver.edu 


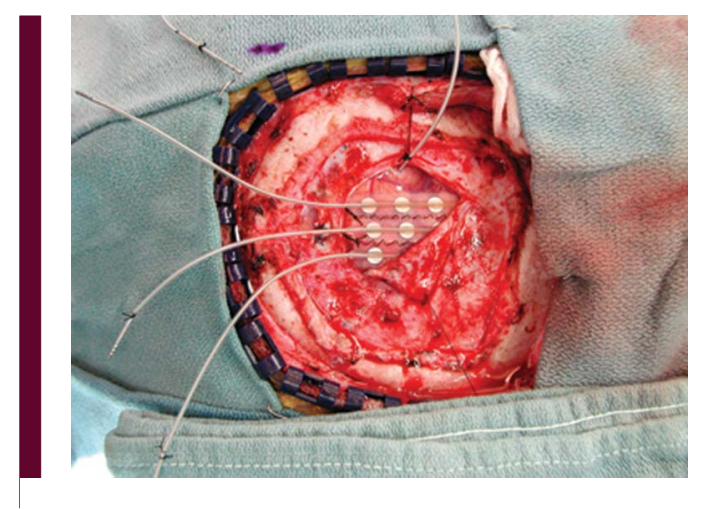

\section{Surgical options for movement disorders have evolved dramatically} over the past half-century, resulting from increasing knowledge of functional neuroanatomic circuitry and progress in medical device technology.

survival. ${ }^{1}$ An emerging approach that appears to allow for intraoperative real-time detection of residual high-grade tumor tissue relies upon preoperative IV delivery of 5-aminolevulinic acid (5-ALA). This biologically inert compound, which is the first component in the porphyrin synthesis pathway, ultimately leads to the accumulation of the fluorescent compound protoporphyrin IX in tumor tissue (due to a paucity of ferrochelatase). The resulting "labeled" tumor can subsequently be visualized using a surgical microscope equipped for blue light emission. The utility of this method for maximizing brain tumor resection was recently explored in a large European multicenter, randomized phase III trial, which confirmed that patients with malignant glioma undergoing fluorescence-guided surgical resection demonstrated less residual enhancing disease on postoperative imaging when compared to patients undergoing standard "white light" guided resection. ${ }^{2}$ This difference was associated with a higher 6-month progression-free survival, although more aggressive resection was also associated with more frequent short-term neurologic decline.

5-ALA is not currently FDA-approved for use in the United States, resulting in widespread use of the compound under the auspices of clinical research protocols. Ongoing experimentation is also focusing on efforts to augment the sensitivity of detection to allow for fluorescence-guided resection of tumors that do not exhibit high degrees of 5-ALA uptake and metabolism. In addition, the potential utility of 5-ALA for photodynamic therapy is also being explored.

\section{Gene therapy for Parkinson disease}

Surgical options for movement disorders have evolved dramatically over the past half-century, resulting from increasing knowledge of functional neuroanatomic circuitry and progress in medical device technology. Early approaches involving lesioning of the globus pallidus and thalamus were irreversible, and these techniques have become more infrequently used in modern times. More recently, development of deep brain stimulation (DBS) in association with stereotactic guidance has allowed for greater elegance in the surgical management of movement disorders. However, in spite of confirmed clinical benefits, DBS often provides incomplete treatment of symptoms associated with nigrostriatal degeneration and does not provide for reversal of the underlying pathology. In addition, the need for permanently implanted hardware is associated with a host of postoperative issues, including infection, hardware malfunction, and limited battery life requiring surgical replacement. Therefore, the ideal surgical strategy for treatment of movement disorders would encompass targeting and reversal of underlying pathologic changes and the restoration of degenerated neural circuitry without the need for implanted hardware.

For these purposes, various strategies of biological restoration have been considered in a range of neurologic diseases. Social and practical concerns have sorely limited ongoing research and clinical use of stem cell-based technology. More recently, the utility of gene therapy has been explored in clinical trials in a wide range of inherited and neurodegenerative disorders, including Parkinson disease. Recently, results have been reported from a phase II randomized, sham-surgery controlled trial investigating the stereotactic delivery of an adenovirus expressing the glutamic acid decarboxylase gene (AAV2-GAD) into the subthalamic nucleus of patients with Parkinson disease. ${ }^{3}$ At baseline, loss of dopaminergic tone from nigrostriatal pathways in 
Figure 1 Imaging in patient with Parkinson disease
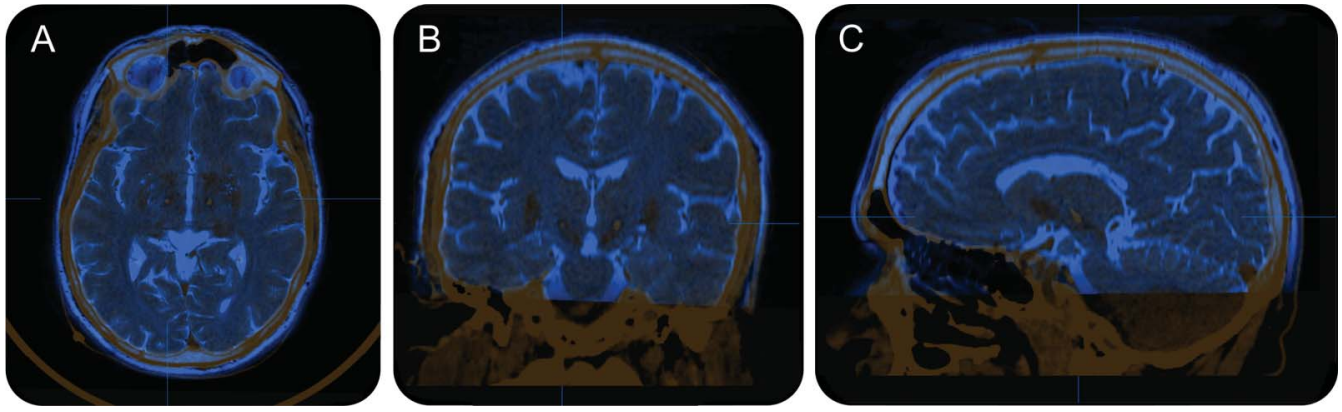

Axial (A), coronal (B), and sagittal (C) preoperative MRI fused with a postoperative CT image in a 56-year-old patient with Parkinson disease who underwent stereotactic bilateral infusion catheter placement into the subthalamic nuclei for delivery of AAV2-GAD.

affected patients results in increased excitatory input from the subthalamic nucleus to the globus pallidus internus, which is the primary inhibitory input to the motor thalamus. GAD is the rate-limiting enzyme in the production of GABA; as such, delivery and activity of AAV2-GAD within the STN could produce increased levels of inhibitory GABAergic potentials and would theoretically provide similar effects to the presumed inhibitory action produced by a deep brain stimulator. Patients randomized to the treatment group underwent stereotactic intraoperative infusion of AAV2-GAD to the subthalamic nucleus (figure 1) while patients randomized to sham-surgery underwent partial thickness burr holes alone. Treated patients showed an improvement of 8.7 points on the Unified Parkinson's Disease Rating Scale (UPDRS) at the 6-month follow-up when compared to controls, which represented a statistically significant clinical improvement, in the absence of significant procedurally related or long-term complication.

Although preliminary, this well-controlled surgical trial further confirmed the safety and tolerability of gene transfer for Parkinson disease. Larger scale clinical trials will be under way soon, and it can be predicted that additional targets for genetic intervention in neurodegenerative disease will be forthcoming.

\section{The artificial disc in treatment of degenerative cervical spine disease}

Treatment of cervical degenerative disc disease has been a major clinical focus for neurosurgeons over many decades. Cloward originally described the surgical approach for anterior cervical discectomy and fusion (ACDF) nearly 50 years ago, and since that time this procedure has become the gold standard for treatment of symptomatic cervical disc disease. However, in longterm follow-up of patients undergoing cervical arthrodesis, it has become apparent that fusion of a dynamic segment of the cervical spine may predispose adjacent levels to more rapid degeneration (figure 2). So-called "adjacent level disease" may lead to new symptoms and necessitate additional surgical procedures in many cases. It has been proposed that more biomechanically faithful fusion hardware may preserve the natural distribution of force throughout the spinal column and therefore prevent worsening degeneration in adjacent levels. To this end, development of artificial discs has been of great interest. The presumed benefit associated with disc replacement is retention of physiologic motion between vertebral bodies, which would be predicted to reduce mechanical forces that are otherwise maximized with rigid bony fusion. The concept of disc replacement, otherwise known as cervical arthroplasty, has existed within the experimental realm since the late 1980s. Since the first experimental results from the Cummins-Bristol disc were made available in the mid-1990s, many advances have occurred in artificial disc technology. More recently, several prospective clinical trials have been designed to perform comparative evaluation of clinical outcomes between artificial cervical disc arthroplasty (ACDA) and standard ACDF. 
Figure 2 Imaging in patient with cervical foraminal stenosis

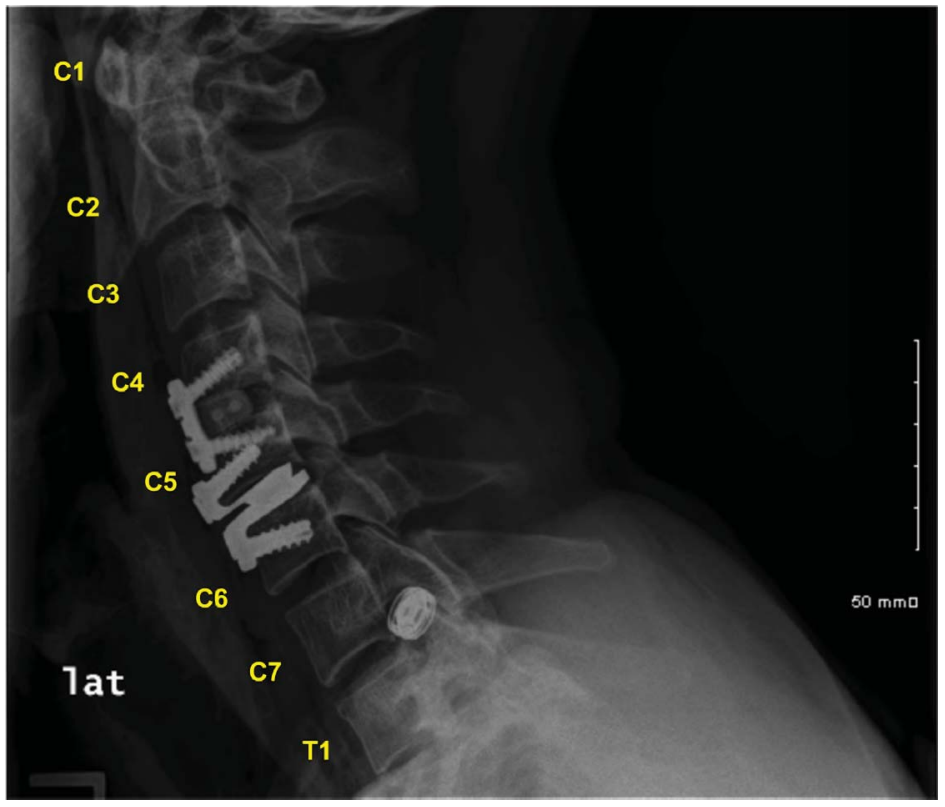

Lateral postoperative plain films from a 40-year-old patient with cervical foraminal stenosis. The images provide detail from a hybrid construct highlighting the radiographic differences between a traditional anterior cervical fusion with cervical arthrodesis (C4-5 level) and cervical arthroplasty with an artificial disc (C5-6 level).

Outcomes have been reported for 2 randomized trials comparing artificial disc technology to standard approaches for ACDF. 4,5 Both of these studies demonstrated acceptable long-term durability of the cervical implant, equipoise in neurologic outcomes, and statistically significant improvement in neck disability index scores. The reoperation rate for adjacent level degeneration or device failure was similar between the ACDA and ACDF groups in both studies, although a nonsignificant trend toward a lower rate of operation at adjacent levels was observed within the ACDA groups. When considering the preservation of cervical motion following ACDA vs ACDF, Sasso et al. ${ }^{4}$ demonstrated a statistically significant increase in the range of motion with flexion and extension at 48 months follow-up in the ACDA cohort, while patients undergoing ACDF experienced a mean decrease in range of motion over a similar time frame.

Although significant opportunities remain for ongoing research, ACDA for single-level symptomatic cervical disc disease appears to show preliminary promise. Future clinical studies and long-term follow-up will likely shed light on additional technological advances in artificial disc design that may result in functional improvement. In addition, further information regarding appropriate patient selection for disc replacement may also significantly augment the efficacy of implanted devices.

\section{Neuromonitoring in acute neurologic disease}

Over 2 million Americans are affected by acute neurologic injury on a yearly basis, and associated yearly costs for medical management of patients with stroke, traumatic brain injury, and intracranial hemorrhage in the United States total in the tens of billions of dollars. Specialized neurologic intensive care units (NICU) under the direction of trained critical care specialists have become increasingly common, and our experience suggests that patients with acute neurologic injury managed within a dedicated NICU setting achieve superior neurologic outcomes when compared with patients who receive their care in generalized surgical and medical intensive care units.

Individuals with acute neurologic disease are often at significant risk for worsening brain injury in the hours to days after the initial damage. Critically, secondary neurologic 


\section{The use of machines to maintain neurologic function in the face of neurodegeneration or profound neurologic injury is a topic of both scientific and metaphysical interest.}

complications are often the result of clinically reversible neurophysiologic changes. Therefore, the timely detection of such changes in brain health is an issue of critical importance for improving patient outcomes. While there have been a variety of devices designed for intracranial implantation and monitoring of various aspects of brain physiology, over the past decade several of these systems have come into more common use in the NICU setting. The gold standard continues to be insertion of fiber-optic intracranial pressure sensors, which are definitively indicated in the monitoring and treatment of patients with severe traumatic brain injury who present with a poor neurologic examination. More recently, development of devices that measure tissue oxygenation (Licox), cerebral perfusion (thermal diffusion flowmetry), and levels of relevant products of cerebral metabolism (microdialysis) have come into more widespread clinical practice. ${ }^{6}$ Our group has recently described the use of intracortical multicontact electrode implantation to provide highfidelity EEG data and sensitive detection of worsening neurologic function in braininjured patients. ${ }^{7}$ Although these newer invasive monitoring systems have yet to gain standard-of-care status, multimodality approaches are being increasingly employed to guide intensive care therapy and will likely show significant benefit for preventing secondary neurologic injury.

\section{Brain-machine interface}

The use of machines to maintain neurologic function in the face of neurodegeneration or profound neurologic injury is a topic of both scientific and metaphysical interest. Brainmachine interfaces (BMI), or "neuroprosthetics," are conceptual devices through which brain electrical signals are recorded, interpreted, and processed in such a way as to produce meaningful output that can be directed at a machine capable of performing a task. The use of BMI has been proposed in patients with stroke, amyotrophic lateral sclerosis, cervical spinal cord injury, optical blindness, and many other conditions where neurologic function may be compromised while some level of cortical processing has been left intact.

Currently, a variety of approaches are being pursued in the development of BMI. Signal acquisition is the first step in the process of producing neuroprosthetic output. Approaches have utilized data recorded from standard scalp EEG electrodes, subdural electrodes, or, more recently, microelectrode arrays implanted into the cerebral cortex. Although extraordinarily detailed data generated by implanted microarrays is attractive for driving BMI, longevity of the brain-electrode interface is often limited due to astrocytic scarring around the implanted electrodes, neuronal cell death, and immune responses leading to signal degradation over time. In addition, the transformation of the extremely dense and complex electrophysiologic data, recorded from microarrays, into meaningful output requires the use of powerful computing platforms by individuals who are experienced in computer programming, signal processing, and bioengineering.

Although there are no widely available clinical options for BMI at this time, there have been early results from several groups that provide proof-of-concept data for the ongoing development of BMI. Donoghue and Hochberg at Brown University have made major contributions to the field over the past decade, primarily focusing on the use of microelectrode array recording from the cortex of nonhuman primates as well as a small number of 


\section{Neurosurgery: Five new things}

- Fluorescence-guided surgery is increasing the extent of brain tumor resection.

- Viral vectors for restorative gene delivery to treat Parkinson disease are being developed and show preliminary efficacy in clinical trials.

Artificial disc implantation to treat cervical spine disease is emerging as an alternative to cervical fusion, with equivalent patient outcomes, while retaining physiologic range of motion.

- Sensitive neuromonitoring technologies are being developed and integrated into the care of the brain-injured patient with the goal of preventing secondary neurologic injury.

Brain-machine interfaces have moved from conceptual devices to active human clinical experimentation and show promise in the control of simple tasks. tetraplegic patients. This group recently reported on the results of microelectrode recording of signals from a silicone microelectrode array implanted in the motor cortex of a tetraplegic human for 1,000 days, confirming a durable brainelectrode interface allowing for signal recording linked to performance of a series of 2-dimensional tasks involving movement of a cursor across a computer screen and then performing a "click" function when the cursor reached the correct target. ${ }^{8}$ Critically, this group also recently reported the ability to drive "reach and grasp" functions by 2 patients implanted within the primary motor cortex with a 96-channel microarray. ${ }^{9}$ In addition to providing output signals to influence the external environment, the ability to provide sensory feedback through BMI has been under active exploration. Nicolelis' group at Duke University recently reported on the results of a "brain-machine-brain" approach that provided microstimulatory sensory cortical feedback data from a robotic actuator driven from signals recorded from the motor cortex of nonhuman primates. ${ }^{10}$ These results, although preliminary, are extremely encouraging and will likely lead to further development of devices designed to interact directly with the human brain and drive subsequent action in the external environment.

\section{CONCLUSIONS}

Ongoing technological advances clearly continue to reshape the spectrum of surgical options for a range of neurologic disorders. Although the aforementioned advances remain within the realm of clinical experimentation at this time, all have passed the "proof-of-principle" barrier and may emerge into mainstream neurosurgical practice in the relatively near future.

\section{REFERENCES}

1. Sanai N, Polley MY, McDermott MW, Parsa AT, Berger MS. An extent of resection threshold for newly diagnosed glioblastomas. J Neurosurg 2011;115:3-8.

2. Stummer W, Pichlmeier U, Meinel T, Wiestler OD, Zanella F, Reulen HJ; ALA-Glioma Study Group. Fluorescence-guided surgery with 5-aminolevulinic acid for resection of malignant glioma: a randomized controlled multicentre phase III trial. Lancet Oncol 2006;7:392-401.

3. LeWitt PA, Rezai AR, Leehey MA, et al. AAV2-GAD gene therapy for advanced Parkinson's disease: a double-blind, sham-surgery controlled, randomised trial. Lancet Neurol 2011;10:309-319.

4. Sasso RC, Anderson PA, Riew KD, Heller JG. Results of cervical arthroplasty compared with anterior discectomy and fusion: four-year clinical outcomes in a prospective, randomized controlled trial. J Bone Joint Surg Am 2011;93:1684-1692.

5. Burkus JK, Haid RW, Traynelis VC, Mummaneni PV. Long-term clinical and radiographic outcomes of cervical disc replacement with the Prestige disc: results from a prospective randomized controlled clinical trial. J Neurosurg Spine 2010;13:308-318.

6. Hemphill JC, Andrews P, De Georgia M. Multimodal monitoring and neurocritical care bioinformatics. Nat Rev Neurol 2011;7:451-460.

7. Waziri A, Claassen J, Stuart RM, et al. Intracortical electroencephalography in acute brain injury. Ann Neurol 2009;66:366-377.

8. Simeral JD, Kim SP, Black MJ, Donoghue JP, Hochberg LR. Neural control of cursor trajectory and click by a human with tetraplegia 1000 days after implant of an intracortical microelectrode array. J Neural Eng 2011;8:025027.

9. Hochberg LR, Bacher D, Jarosiewicz B, et al. Reach and grasp by people with tetraplegia using a neurally controlled robotic arm. Nature 2012;485:372-375. 
10. O'Doherty JE, Lebedev MA, Ifft PJ, et al. Active tactile exploration using a brain-machine-brain interface. Nature 2011;479:228-231.

\section{DISCLOSURES}

The authors report no disclosures relevant to the manuscript. Go to Neurology.org/cp for full disclosures.

\section{Related articles from other AAN physician and patient resources}

Neurology ${ }^{\circledR} \bullet \quad$ www.neurology.org

Epilepsy surgery trends in the United States, 1990-2008 April 17, 2012; 78: 1200-1206.

Neurology Now ${ }^{\circledR}$

- www.neurologynow.com

It's Electric!! Vagus nerve stimulation may improve uncontrolled epileptic seizures July/August 2009; 34-35.

\section{Continuum: Lifelong Learning in Neurology ${ }^{\circledR} \quad$ www.an.com/continuum}

Emerging therapies

December 2008; 14:80-97.

Neurology Today ${ }^{\circledR}$ - www.neurotodayonline.com

PD Patients Continue to Benefit from DBS After a Decade

May 19, 2011; 11:1, 13. 